\title{
An analysis of cross-correlations in an emerging market
}

\author{
Diane Wilcox ${ }^{\mathrm{a}, 1}$ Tim Gebbie $^{\mathrm{b}}$ \\ ${ }^{a}$ Dept. of Mathematics 85 Applied Mathematics, University of Cape Town, \\ Rondebosch, 7700, South Africa \\ ${ }^{\mathrm{b}}$ Futuregrowth Asset Management, Private Bag X6, Newlands, 7725, South Africa
}

\begin{abstract}
We apply random matrix theory to compare correlation matrix estimators $C$ obtained from emerging market data. The correlation matrices are constructed from 10 years of daily data for stocks listed on the Johannesburg Stock Exchange (JSE) from January 1993 to December 2002. We test the spectral properties of $C$ against random matrix predictions and find some agreement between the distributions of eigenvalues, nearest neighbour spacings, distributions of eigenvector components and the inverse participation ratios for eigenvectors. We show that interpolating both missing data and illiquid trading days with a zero-order hold increases agreement with RMT predictions. For the more realistic estimation of correlations in an emerging market, we suggest a pairwise measured-data correlation matrix. For the data set used, this approach suggests greater temporal stability for the leading eigenvectors. An interpretation of eigenvectors in terms of trading strategies is given, as opposed to classification by economic sectors.
\end{abstract}

Key words: Random matrices, Cross-correlations, Finance, Emerging markets PACS: 02.10.Yn, 05.40.Ca, 05.45.Tp, 87.23.Ge

\section{Introduction}

Correlation matrices are common to problems involving complex interactions and the extraction of information from series of measured data. Our aim is to determine empirical correlations in price fluctuations of daily sampled price data of distinct shares in a reliable way. Our investigation is based on 10 years of daily data for 250-350 traded shares listed on the JSE Main Board from January 1993 to Dec 2002.

Email addresses: diane@maths.uct.ac.za (Diane Wilcox), tim.gebbie@physics.org (Tim Gebbie).

1 Corresponding author. 
There are several aspects to the question of how to calculate correlations in financial time series. In particular, missing data and thin trading (no prices changes for a stock over several time periods) may be significant. Random correlations in price changes are likely to arise in an ensemble of several shares. Furthermore, for a portfolio of $N$ distinct assets, there will be $N(N-1) / 2$ entries in a correlation matrix which has been determined from time series of length $L$. When $L$ is not large, the calculated covariance matrix may be dominated by measurement noise. Hence, it is necessary to understand effects of (i) noise (ii) finiteness of time series (iii) missing data and (iv) thin trading in determination of empirical correlation.

The properties of random matrices first became known with Wigner's seminal work in the 1950's for application in nuclear physics in the study of statistical behaviour of neutron resonances and other complex systems of interactions ([23], [5] and [14]). More recently random matrix theory has been applied to calibrate and reduce the effects of noise in financial time series and to investigate constraints on rational (empirically based) decision making (cf. [13], [17], [15], [28], [8] [26], [10], [9] ). Correlation matrices are computed for the data under investigation and quantities associated with these matrices may be compared to those of random matrices. The extent to which properties of the correlation matrices deviate from random matrix predications clarifies the status of the information derived from the computation of covariances. In several studies of shares traded in the S\&P 500 and DAX, it was found that, aside from a small number of leading eigenvalues, the eigenvalue spectra for the measured data coincide with theoretic random matrix predictions, i.e. it was found that the estimation of covariances is dominated by random noise. In [24], postulated a model for the correlations which explained the observed spectral properties. RMT has also been shown to yield an improved estimation technique: an estimated correlation matrix can be filtered by removing the contributions of eigenvalues which lie in the RMT range. In [25] it is shown that noise levels in the correlation matrix depend on the ratio $N: L$, where $N$ denotes the number of stocks and $L$ denotes the length of the time-series.

\subsection{Correlation matrices and missing data in an emerging market}

In this paper we consider the problems of missing data and thin trading in determination of empirical correlation in daily sampled price fluctuations. We analyze the data base containing prices $S_{i}(t)$, the prices of assets $i=1, \ldots, N$ at time $t$ as follows. We first find the change in asset prices

$$
r_{i}(t)=\ln S_{i}(t+\triangle t)-\ln S_{i}(t) .
$$

The usual cross-correlation matrix for idealized data (non-zero price fluctuations and no missing data) is given by 


$$
C_{i j}:=\frac{\left\langle r_{i} r_{j}\right\rangle-\left\langle r_{i}\right\rangle\left\langle r_{i}\right\rangle}{\sigma_{i} \sigma_{j}}
$$

where $\langle\ldots\rangle$ denotes average over period studied and $\sigma_{i}^{2}:=\left\langle r_{i}^{2}\right\rangle-\left\langle r_{i}\right\rangle^{2}$ is the variance of the price changes of asset $i$. Alternatively one could write

$$
C_{i j}=\frac{1}{L} \sum_{t=1}^{L} R_{i}(t) R_{j}(t)
$$

where $L$ denotes the uniform length of the time series and $R_{i}(t)$ denotes the price change of asset $i$ at time $t$ such that the average values of the $R_{i}^{\prime} s$ have been subtracted off and the $R_{i}^{\prime} s$ are rescaled so that they all have constant volatility $\sigma_{i}^{2}:=\left\langle R_{i}^{2}\right\rangle=1$. This is written as $\mathbf{C}=\frac{1}{L} \mathbf{M} \mathbf{M}^{T}$ where $\mathbf{M}$ is a $N \times L$ matrix and $\mathbf{M}^{T}$ is its transpose (cf. [17]).

The pairwise measured-data cross-correlation matrix using the pairwise deletion method [33], [34]) for the case when there is missing data in time series of returns is computed as follows:

$$
\mathcal{C}_{i j}:=\frac{\left\langle\rho_{i} \rho_{j}\right\rangle-\left\langle\rho_{i}\right\rangle\left\langle\rho_{i}\right\rangle}{\sigma_{i} \sigma_{j}}
$$

where $\rho_{i}$ and $\rho_{j}$ denote subseries of $r_{i}$ and $r_{j}$ such that there exists measured data for both $\rho_{i}$ and $\rho_{j}$ at every time period in the subseries, and $\langle\ldots\rangle$ denotes average over period studied, $\sigma_{i}^{2}:=\left\langle\rho_{i}^{2}\right\rangle-\left\langle\rho_{i}\right\rangle^{2}$ is the variance of the price changes of asset $i$.

\section{Random Matrix Theory (RMT) predictions}

We summarise four known universal properties of random matrices, namely the Wishart distribution for eigenvalues, the Wigner surmise for eigenvalue spacing, the distribution of eigenvector components and the inverse participation ratio for eigenvector components, which will be applied in our analysis.

Let $A$ denote an $N \times L$ matrix whose entries are i.i.d random variables which are normally distributed with zero mean and unit variance. As $N, L \rightarrow \infty$ and while $Q=L / N$ is kept fixed, the probability density function for the eigenvalues of the Wishart matrix (or Laguerre ensemble) $R=\frac{1}{L} A A^{T}$ is given by ([1], [11], [31]):

$$
p(\lambda)=\frac{Q}{2 \pi} \frac{\sqrt{\left(\lambda_{\max }-\lambda\right)\left(\lambda-\lambda_{\min }\right)}}{\lambda}
$$

for $\lambda$ such that $\lambda_{\min } \leq \lambda \leq \lambda_{\max }$, where $\lambda_{\min }$ and $\lambda_{\max }$ satisfy 


$$
\lambda_{\max / \min }=1+\frac{1}{Q} \pm 2 \sqrt{1 / Q}
$$

The distribution of eigenvalue spacings was introduced as a further test for the case when the empirical eigenvalue distribution does not deviate significantly from the RMT predication. The so-called Wigner surmise for eigenvalue spacings [23] is given by

$$
p(s)=\frac{s}{2 \pi} \exp \left(-\frac{s \pi^{2}}{4}\right),
$$

where $s=\left(\lambda_{i+1}-\lambda_{i}\right) / d$ and $d$ denotes the average of the differences $\lambda_{i+1}-\lambda_{i}$ as $i$ varies 2 .

It has been found that the eigenvector components $v_{a}^{i}$ for $a=1 \ldots n$ of an eigenvector $\mathbf{v}_{a}$ are normally distributed with zero mean and unit variance [14], [4],

$$
p(u)=\frac{1}{\sqrt{2 \pi}} \exp \left(-\frac{u^{2}}{2}\right)
$$

The inverse participation ratio (IPR) is used to analyze the structure of the eigenvectors of the correlation matrix [28]. The $i$ th component $v_{a}^{i}$ of $\mathbf{v}_{a}$ corresponds to the contribution of the $i$ th time series to that eigenvector. To quantify this contribution, the IPR for $\mathbf{v}_{a}$ is defined

$$
I_{a}=\sum_{i=1}^{N}\left(v_{a}^{i}\right)^{4},
$$

where $N$ is the number of time series (the number of shares) and, hence, number of eigenvalue components. If the components of the eigenvector are identical, $v_{a}^{i}=\frac{1}{\sqrt{N}}$, then $I_{a}=1 / N$; if there is only a single non-zero component, then $I_{a}=1$. In general, the IPR is a reciprocal of the number of eigenvector components which are contribute significantly, i.e. which are different from zero. It is found that $E\left[I_{a}\right]=3 / N$ since the kurtosis for the distribution of eigenvector components is 3 .

\section{Analysis of Johannesburg Stock Exchange data}

The JSE is one of the 20 largest national stock markets in the world. We summarise some of its known qualitative features. Although many of the main board JSE shares are illiquid, the market as a whole is a fairly liquid one. There is share concentration in half-dozen shares: these dominant shares account for almost a third of the index and have

$\overline{2}$ Unfolded eigenvalues are used in practice [5], [14] [28]. 
a large bias towards resources. The resources sector in turn is strongly correlated with the dollar-rand exchange rate, an exogenous factor that has a dominant influence on price dynamics in South African stock markets. Next it is noteworthy that different shares are listed on the JSE at different times and, hence, different shares do not always trade on the same day. However, some shares which do not trade often may occasionally trade in large volumes for several days. These realities exacerbate the problem of estimating correlations in a reliable way.

The data set used in this study incorporated a zero-order hold for prices when there was no trading. This approach accounts for sequences of zero-valued returns in the return timesseries even though no measurements occurred. While it has often been convenient to set the returns to zero in the periods preceding listing of shares to avoid data holes, in general, this strategy seems to give rise to a significant gaussian component to estimated correlations. We investigate the effect of various treatments and interpretations of measurements in the context of price time-series. The approach here favours the notions that (1) if no price was discovered for a given share then there was no measurement, and (2) share cross-correlations can only be computed when there are measurements on the same day.

\subsection{Filtering and partitioning the data}

The data set of 10 years of data from 1 January 1993 to 31 December 2002 was split into annual epochs. The data was windowed to create 6 sets of 5 years of daily price data. Each block was screened to remove shares that were de-listed or which traded quite infrequently. For each year in a given epoch of 5 years, this was achieved by dropping all shares that neither recorded price measurements at year-end nor traded at least once in the preceding month. Table 1 gives the data sets used.

In Figure 1 we reconstruct price indices (not total price) from the market capitalization of each individual stock based on the economic sector membership, i.e. a weight in a particular index would be the stocks market capitalization divided by the total portfolio market capitalization. The reason for this is that there is no complete constituent history available over for the full 10 year period studied - the indices were reconstructed by the authors. This also ensures consistency between the indices provided and the stocks and the stock data used in the study. Figure 1 corroborates evidence of negative correlation which is presented when we consider temporal stability of our results in Subsection 3.4 below - the dominance of the financial sector peaks in 1998 for the period under investigation; thereafter the resources sector begins to dominate the market.

\subsection{Three estimates of cross-correlations}

We investigate correlation structure by considering correlation matrices of the data sets

in Table 1 in three different ways. Case 1: we assign the value of zero whenever there is no measured data for a return $r_{i}(t)$ for asset $i$ at time $t$; we then compute the correlation 


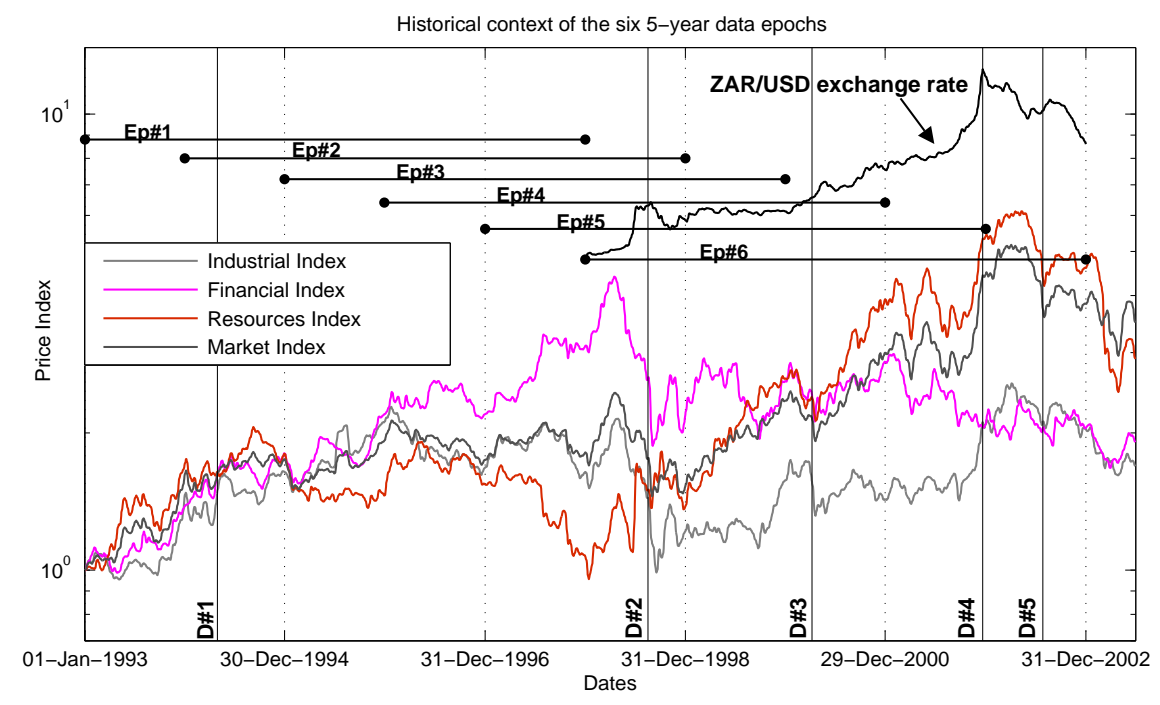

Fig. 1. Historical context of price evolution for the period studied: Indices are reconstructed using the market capitalization of stocks in the industrial, financial and resources sectors as well as the entire market for the market index. The horizontal lines labelled EP\#1, EP\#2 to EP\#6 demarcate time windows 1993-1997, 1994-1998 to 1998-2002, respectively. Specific dates are highlighted by vertical lines labelled: D\#1 - 27 Apr 1994 - the first SA elections of the post-Apartheid era, D\#2 - 17 Aug 1997 - Russian GKO default, D\#3 - 10 Apr 2000 - proxy date for Nasdaq crash, D\#4 - 20 Dec 2001 - SA Rand (ZAR) crash D\#5 - 27 Jul 2002 Sarbanes-Oxley Act. Inset: The ZAR/USD exchange rate evolution from Jan 1998 to Dec 2002.

matrix in the usual way as described in the introduction. Case 2: we compute the pairwise measured-data correlation matrix to overcome missing measurements. Case 3: we address the problem of no trading, i.e. zero price fluctuations for several time periods in succession. To do so, in the event of 2 or more successive zero-valued price fluctuations we delete the measured return value $r_{i}(t)=0$, effectively turning the zero-valued information into missing data. This compensates for interpolated prices being mistaken for measurements. We then compute the pairwise measured-data correlation matrix.

We considered the problem of non-positive definiteness (this property is destroyed by most missing-data methods, including the one which we implement) by applying the area-minimizing algorithm of [7] to make the matrices in Case 3 positive semi-definite (see also [19]). Lastly, as a further case, we removed bias from the data as a means of removing the market mode (cf. [28]). Details for these cases are not included since they do not add to our discussion on the phenomenology on missing data in this paper.

We note that there are several other methods for treating missing data (see for example [33] and [34]). Our view is that the pairwise deletion method offers a sufficiently robust correlation estimate for the purposes of this analysis of daily data. Listwise deletion of an entire day's records for a day on which there is missing data for a single stock is likely to delete useful data for remaining stocks and results in too few remaining records. Mean substitution and imputation by regression are likely to introduce spurious correlations between stocks which are not listed or which do not trade for several successive periods this is borne out by the comparison of results between Cases 2 and 3. For the application 

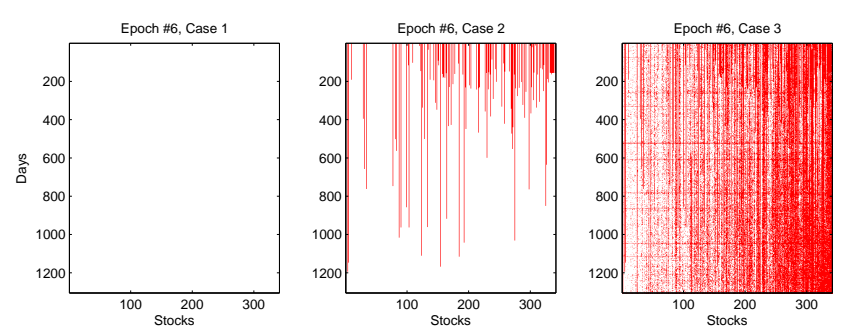

Fig. 2. Missing data is depicted in red, while reasonable data is white space. The three graphs are, from left to right are: Case 1, which includes zero-padding and zero-order hold, Case 2 which has only zero-order hold, and Case 3 which has measured data only. These demonstrate the extent of missing data. The graph reflects daily data from Epoch 6 (1998-2002).
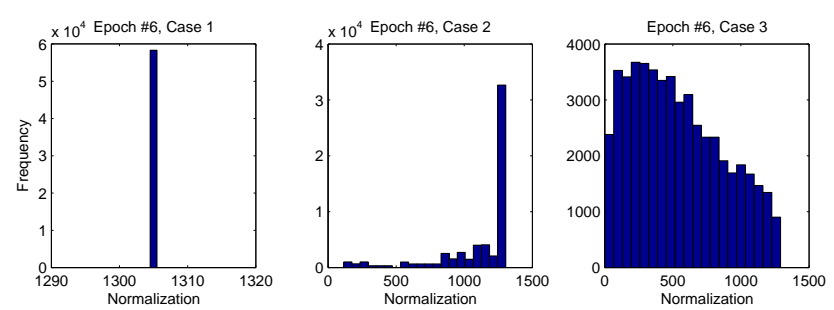

Fig. 3. Frequency distributions of normalisations used in the computation of pairwise correlations are plotted as a histogram for each Case considered in Figure 2. See also Table 1.

of hot deck imputation it is not at all clear which strings of return data it would be appropriate to draw from over the varying time windows. The most promising alternative missing data method would be the use of an expectation maximization algorithm and this is left for further investigation.

The different treatments of the data have significant impact on the relationship between the number of meaningful data points and the normalisation factors used in the computations.

Figure 2 illustrates the occurrence of missing data. In Case 1, zero padding fills all the gaps so there appears to to be no missing data. In Case 2, there is missing data for shares which were not listed at the start of the epoch and constant prices are recorded when no trading has taken place. In case 3, we remove prices when no trading has occurred. Stocks are ordered by market capitalization from left to right. The concentration of red on the right of the (c) is clear evidence that smallest cap stocks tend to trade quite infrequently. Horizontal bands indicate public holidays. That zero order hold was used to interpolate missing data on public holidays within the data set obtained, is a prime example of how measurement error can contaminate a database.

Figure 3 plots the frequency of the normalisation factors used to compute entries in the correlation matrices. For cases 2 and 3, incorrect normalisation factors for (truncated) pairwise matched timeseries would have the effect of distorting correlation estimates when there is missing data. In Case 1, the normalisation factor is the same for all pairs, i.e. it is equal to $L \approx 1305$, the total number of official trading days in the 5 -yr epoch (see table). In Case 2, the normalisation factor is often much larger than the number of days for which shares are actually traded. Here, normalisations in the order of $L$ were frequently used 
Table 1

The data sets used comprising shares traded on the Johannesburg Stock exchange. Each data set starts on the 1 January of the starting year and ends on the 31 December of the ending year.

\begin{tabular}{llcccccc}
\hline & Data set (Epoch) & 1 & 2 & 3 & 4 & 5 & 6 \\
\hline Start date (1 Jan) & 1993 & 1994 & 1995 & 1996 & 1997 & 1998 \\
& End date (31 Dec) & 1997 & 1998 & 1999 & 2000 & 2001 & 2002 \\
& Total no. of shares (N) & 253 & 296 & 321 & 330 & 336 & 341 \\
& No. of trading days (L) & 1304 & 1305 & 1306 & 1306 & 1305 & 1305 \\
\hline \multirow{2}{*}{ Case 1 } & No. of shares used & 253 & 296 & 321 & 330 & 336 & 341 \\
& \% zero returns & $73 \%$ & $70 \%$ & $63 \%$ & $58 \%$ & $53 \%$ & $54 \%$ \\
& \% missing data & 0 & 0 & 0 & 0 & 0 & 0 \\
\hline \multirow{2}{*}{ Case 2 2 of shares used } & 253 & 296 & 321 & 330 & 336 & 341 \\
& \% zero returns & $54 \%$ & $44 \%$ & $41 \%$ & $40 \%$ & $42 \%$ & $46 \%$ \\
& No. of shares used & 244 & 282 & 308 & 310 & 316 & 319 \\
Case 3 3 zero returns & $12 \%$ & $11 \%$ & $12 \%$ & $14 \%$ & $14 \%$ & $15 \%$ \\
& \% missing data & $59 \%$ & $56 \%$ & $50 \%$ & $42 \%$ & $38 \%$ & $36 \%$ \\
\hline
\end{tabular}

even though there was substantially less measured price data. In Case 3, normalisation factors varied from less than 60 up to $L$, with normalisations of 330-390 occurring most frequently.

\subsection{Spectral properties and comparisons with RMT predictions}

In [17], investigating daily price fluctuations for $\mathrm{N}=406$ stocks of the $\mathrm{S} \& \mathrm{P} 500$ for $\mathrm{L}=1309$ days during 1991-1996, with $\mathrm{Q}=3.22$, it was found that the leading eigenvalue was $\approx 25$ times greater than the RMT predicted $\lambda_{\max }$. Adjusting for the total variance $\sigma_{2}$ of the price fluctuations, it was found that $94 \%$ of the spectrum could be attributed to random noise. In [27], the high-frequency TAQ database published by NYSE for the period 1994-1995 was analysed: using 30 min returns for $\mathrm{N}=1000$ companies with $\mathrm{L}=6448$, it was found again that the leading eigenvalue was $\approx 25$ times larger than the RMT predicted value for $\lambda_{\max }=1.94$ and that $\approx 98 \%$ of the eigenvalues could be accounted for as random noise effects. Results of [27] were corroborated in the more extensive study [28] of the same high-frequency TAQ data together with CRSP databases of daily data for common stocks in the NYSE beginning 1925, the AMEX beginning 1962 and the NASDAQ beginning 1972. Investigation of further universal properties confirmed that for eigenvalues within the Wishart range and their corresponding eigenvectors: (a) the distribution of nearest-neighbour spacings were in good agreement with Gaussian Orthogonal Ensemble 
(a) Eigenvalue PDF
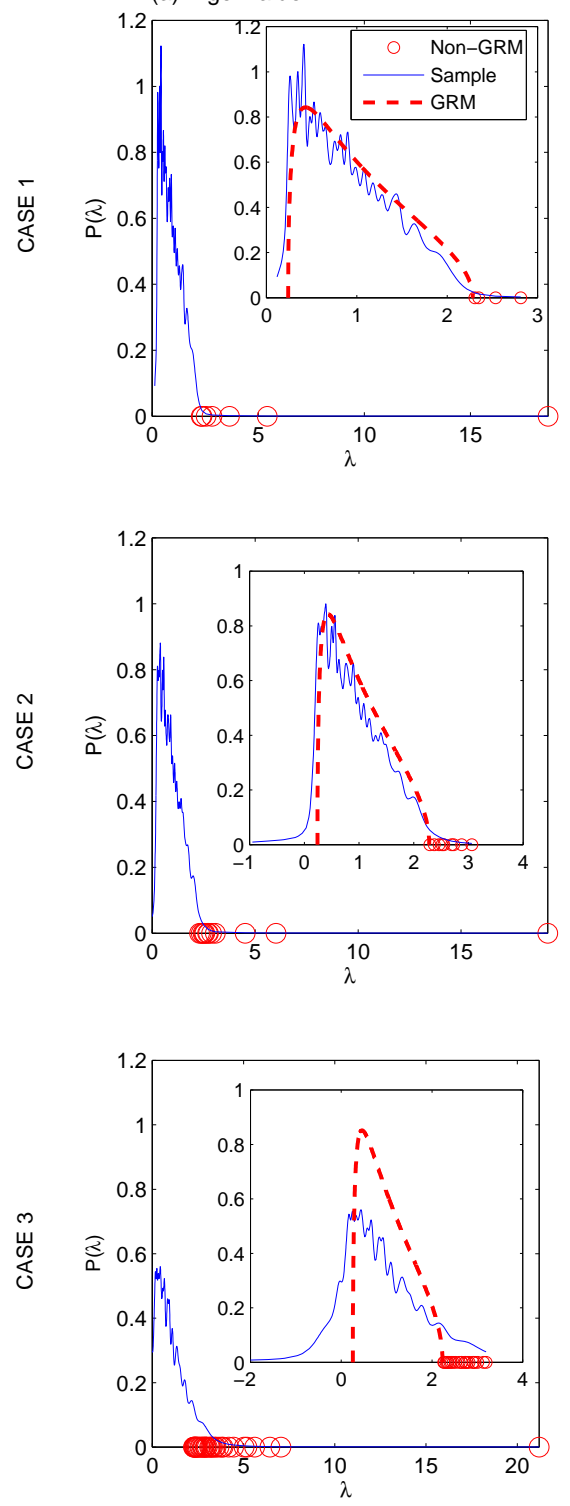

(b) Nearest Neighbour (unfolded)
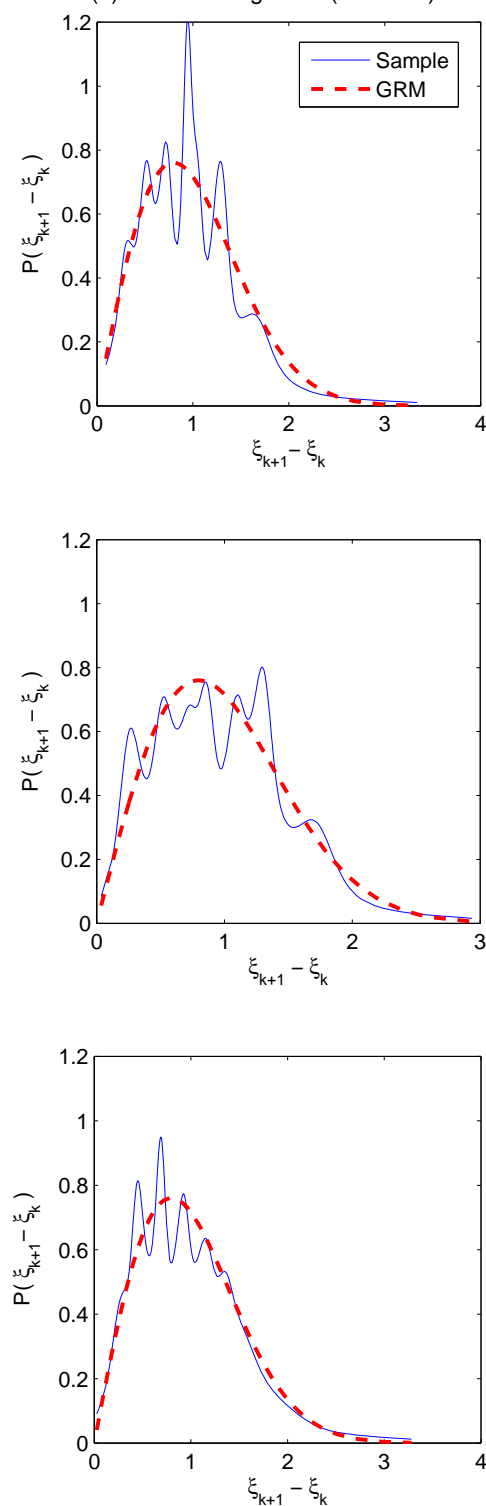

Fig. 4. Daily price returns for JSE main board shares for years 1998-2002 are used to investigate eigenvalue structures of three estimated correlation matrices. Figure 4 (a) shows the eigenvalue density functions with the distinct eigenvalues greater than the maximum RMT predicted value for the same Q-factor as the sample. Insets: plots of the Wishart distribution (Eqn. 1) are superimposed on plots of the small eigenvalues. Figure 4 (b) shows the nearest-neighbour distributions of the folded eigenvalues. Superimposed on these are plots of the Wigner Surmise ( Eqn. 3). The folded eigenvalues were computed using Gaussian broadening and numerical integration.

predictions, (b) the distribution of eigenvector components conformed with the predicted Gaussian distribution with zero mean and unit variance and (c) almost all the eigenvector components contributed equally to the inverse participation ratio except for eigenvectors corresponding to eigenvalues outside the RMT bounds. In the latter cases it was found that almost all stocks participated in the largest eigenvector while for the remaining large eigenvectors there was localization, i.e. only a few stocks contributed to them. Similar 
analysis was conducted on high-frequency data for the DAX for the period Nov 1997 to Dec 1998 to examine intraday dynamics and memory effects in the index [9].

In this section we investigate the same properties in the context of an emerging market. Figure 4 gives a comparison of the eigenvalue densities and nearest-neighbour spacings for the three different cases considered for the last epoch, 1998-2002. It is clear from the graph that most of the eigenvalues for Case 1 are within the range of the Wishart distribution (Eqn. 1). For Case 2, the number of eigenvalues within the noise range is slightly reduced. Some of the eigenvalues are negative in this case. For Case 3 there is a more significant drop in number of eigenvalues in the noise band compared to Case 1; there are also more negative eigenvalues in Case 3 compared to Case 2. In all Cases the nearest-neighbour spacings indicate some agreement with the Wigner surmise (Eqn. 3).

It is clear from the presence of negative eigenvalues in Cases 2 and 3 that the matrices obtained are no longer positive definite. There are several algorithms to obtain positive definite matrices from a non-positive definite estimate [7], [19]. A thorough comparison of these methods (including their impact on eigenvalue distributions and temporal stability) is a separate topic of investigation.

For Case 3 we found that $\approx 88 \%$ of the eigenvalues (including negative values) fell below $\lambda_{\max }=2.23$ (a smaller percentage than in [17], [27] and [28]) and that the largest eigenvalue, $\lambda=21.20$, was $\approx 9.5$ times great than $\lambda_{\max }$ (significantly less than results for developed markets [17], [27] and [28]). The high percentage of eigenvalues below $\lambda_{\min }$ may be attributed to the fact that many of the less liquid stocks behave independently relative to the rest of the market. While a null-hypothesis of Gaussian returns is useful for identifying how zero-padding and zero-order hold add noise to data, it is possible that the noise range $\left[\lambda_{\max }, \lambda_{\text {min }}\right]$ is wider than suggested by equation (1). Simulations for time-series with Gaussian returns populated appropriately with missing data yield almost identical distributions of eigenvalues as the Wishart distribution. Different stocks in the SA market exhibit a range of return phenomenologies, including periodic and aperiodic behaviour [32]; hence, the construction of a representative null-hypothesis becomes problematic. From qualitative information about the market, results for inverse participation ratios, temporal stability and style characteristics (discussed in sections below) and by an analysis of the dimensionality of the SA market [29], a more realistic estimate seems to be that 8-9 eigenvalues are associated with information content $(\approx 2 \%$ of the total).

Figure 5 (a) gives the distributions of component values for eigenvectors corresponding to the $1^{\text {st }}, 2^{\text {nd }}, 3^{\text {rd }}, 10^{\text {th }}$ and $100^{\text {th }}$ largest eigenvalues for the last epoch, 1998-2002. In all three cases the first three distributions deviate significantly from the Porter-Thomas null-hypothesis (Eqn. 4); the distributions corresponding to smaller eigenvalues are in greater agreement with their random matrix counterparts. In Case 3, the components for the leading eigenvector are mostly positive valued (as in [17], [27] and [28]).

Figure 5 (b) gives the inverse participation ratios (IPR's) plotted against corresponding eigenvalues. In all three cases, the IPR for the leading eigenvector is approximately equal to the RMT prediction of 3/N (Eqn. 5), indicating contributions from almost all stocks in 
Table 2

Average percentage of variance explained by the leading eigenvalues (average taken over 6 epochs).

\begin{tabular}{lccc}
\hline & Case 1 & Case 2 & Case 3 \\
\hline Total variance & & & \\
$\%$ explained by eigenvalues $1-5$ & $27 \% \pm 5 \%$ & $25 \% \pm 4 \%$ & $28 \% \pm 4 \%$ \\
$\%$ explained by eigenvalues $1-15$ & $43 \% \pm 5 \%$ & $41 \% \pm 4 \%$ & $50 \% \pm 4 \%$ \\
\hline Trace of Correlation matrix & & & \\
$\%$ explained by eigenvalues $1-5$ & $11 \% \pm 1 \%$ & $12 \% \pm 1 \%$ & $15 \% \pm 1 \%$ \\
$\%$ explained by eigenvalues $1-15$ & $18 \% \pm 2 \%$ & $20 \% \pm 2 \%$ & $25 \% \pm 2 \%$ \\
\hline
\end{tabular}

the market. This is consistent with findings in [28]. For Case 1, the IPR's for the $2^{\text {nd }}$ and $3^{\text {rd }}$ largest and the 7 smallest eigenvalues deviate significantly from the random matrix null case. For Cases 2 and 3, the IPR's for largest 6 and 9 eigenvalues, respectively, deviate significantly from the random matrix null case, indicating contributions from only a few stocks (as in [28]); the same is true for several of the smallest eigenvalues for Case 2; for Cases 2 and 3, the rest of the eigenvalues, the mean IPR is greater than the RMT prediction of $3 / \mathrm{N}$ and IPR values fluctuate with greater variance about this mean compared with variance for the null case.

\subsection{Temporal stability of the correlation matrices}

Temporal stabilities of the matrices were investigated for annual variation. In [28], it was found that the largest four eigenvectors obtained from high-frequency data (returns at 30-min intervals) were stable up to time-lags of 1 year, while the largest two eigenvectors from 30 years of daily data were stable for time-scales up to 20 years.

In this investigation, we computed overlap matrices with entries given by estimated correlations between the leading eigenvectors from the last 5-yr epoch, $1998-2002$, with their analogues from the preceding epochs, 1997 - 2001 to 1993 - 1997, as follows: for each epoch, the eigenvectors corresponding to the 15 largest eigenvalues were chosen; each eigenvector was expanded to include components for every share present in the epochs being compared and when a share was not included in one of the epochs, a value of zero was assigned ${ }^{3}$. We let $U(E)$ denote the $N \times 15$ matrices whose columns are the leading 15 eigenvectors from the $E^{\text {th }}$ epoch, $E=6$ for $1998-2002, E=5$ for $1997-2001$, etc. The overlap matrices are hence given by $O(t, \tau)=U(t)^{T} U(t-\tau)$, where $t$ denotes an epoch and $\tau$ denotes a lag in years.

Figure 6 gives an indication of the temporal stability of the eigenvectors associated with the largest eigenvalues for Cases 1 and 3 .

$\overline{3}$ This takes into consideration the fact that each epoch is comprised of slightly different collections of shares 

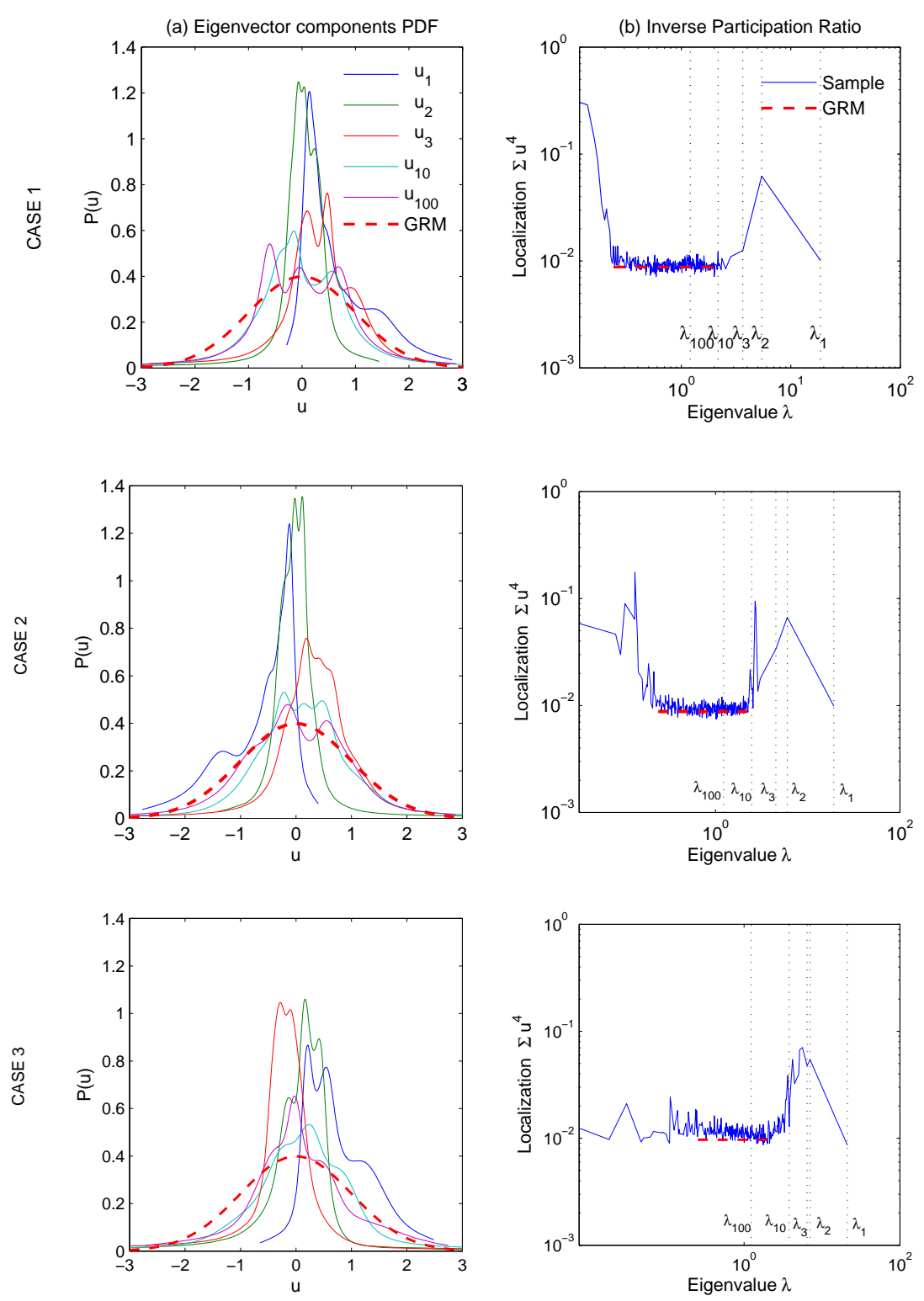

Fig. 5. Daily price returns for JSE main board shares for years 1998-2002 are used to investigate eigenvectors of three estimated correlation matrices. Figure 5 (a) gives the distributions of component values for eigenvectors corresponding to the $1^{\text {st }}, 2^{\text {nd }}, 3^{\text {rd }}, 10^{\text {th }}$ and $100^{\text {th }}$ largest eigenvalues. Plots of the Porter-Thomas distribution (Eqn. 4) are superimposed. Figure 5 (b) gives plots of the inverse participation ratios for the three cases together with the RMT prediction, $E\left[I_{a}\right]=3 / N$, using kurtosis of Eqn.4.

In Case 1 the correlation of the eigenvectors corresponding to the largest eigenvalue is 1 for all lags; the correlation of the eigenvectors corresponding to the second eigenvalues is negative for lags 1 to 4 and the correlation of the eigenvectors corresponding to the third eigenvalues is positive for lags 1 to 4 .

In Case 3, temporal correlations of the eigenvectors corresponding to the largest eigenvalue is alternately positive then negative for successive lags; correlations for the second eigenvector are positive except for time lags of 2 and 5 years where there are no correla- 

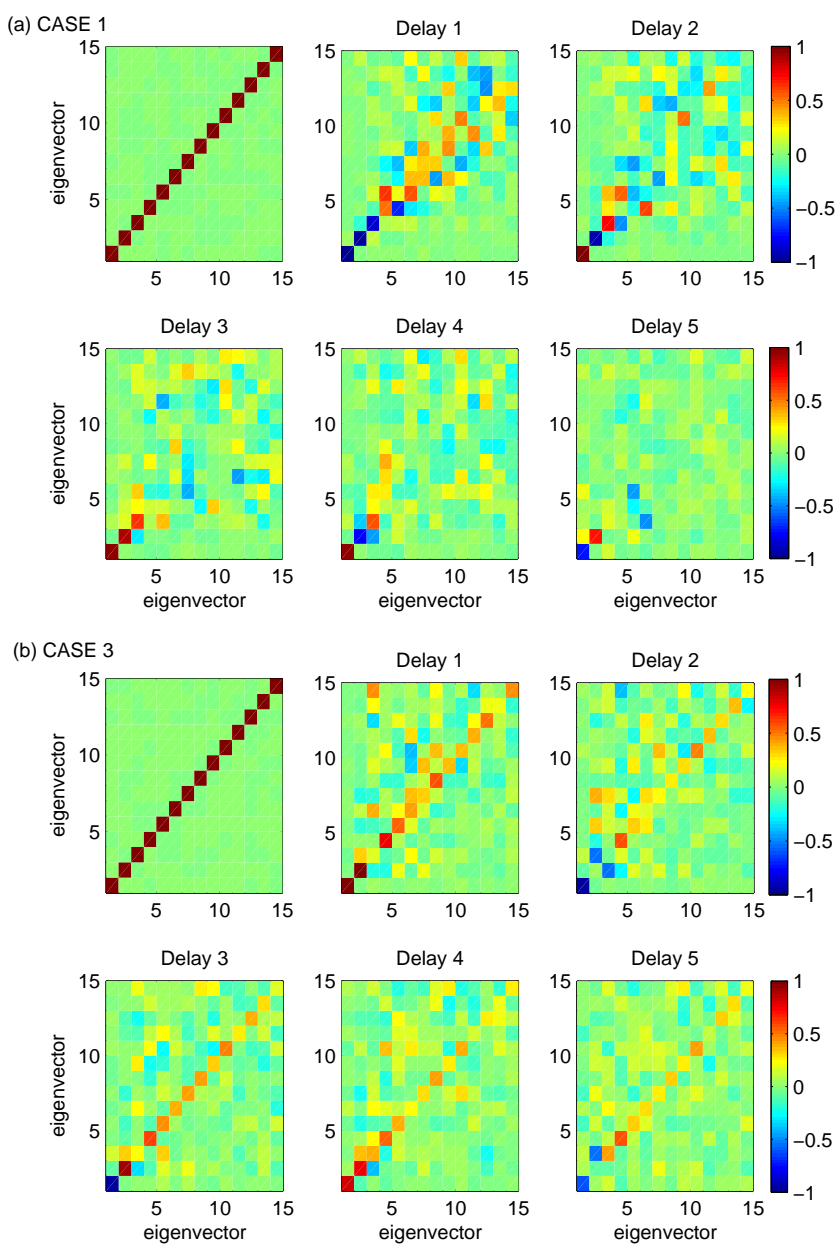

Fig. 6. Overlap matrices are computed using 6 sets of 5-yr epochs of daily returns. Figure 6 (a) and (b) give results for Cases 1 and 3, respectively. The graphs depict estimated correlations between the 15 leading eigenvectors from the last epoch, 1998 - 2002, with their analogues from the preceding epochs, 1998 - 2002 (lag 0), 1997 - 2001 (lag 1) to $1993-1997$ (lag 5).

tions; correlations for the third eigenvalue are insignificant at delays of 1 and 2 years, then tend to be positive for delays of 3-5 years; correlations between eigenvectors corresponding to the next seven eigenvalues tend to be positive for lags 1 to 5 years. The negative correlations in Case 3 are consistent with the negative correlation between the financial and resources sectors and the switch in performance of corresponding indices following the market stresses in emerging markets in the $2^{\text {nd }}$ half of 1998 (cf. Figure 1). This case offers the greatest evidence of temporal stability. The overlap matrices are all computed against Epoch number 6, 1998-2002, where the market is also influenced by the crash of the Rand in 2001 (cf. ZAR/USD exchange rate inset in Figure 1).

There is also evidence of stability for Case 2 - in this case the results are similar to but slighter weaker than those for Case 3 . 

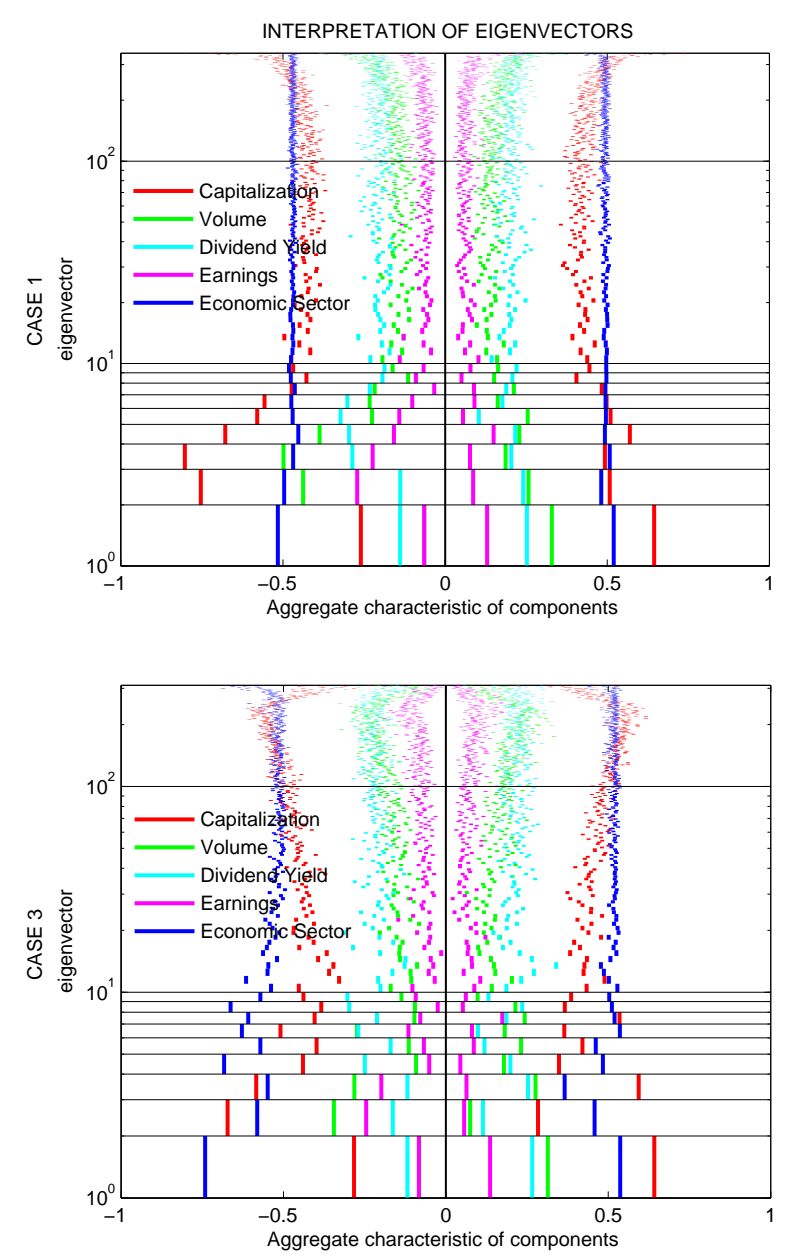

Fig. 7. Fundamental characterizations of components of the eigenvectors for Cases 1 and 3 for 1998-2002 are given in Figure 7 (a) and (b), respectively. Characteristic spectra are plotted in increasing order: the lowest band represents the spectrum for the first eigenvector, the next spectrum corresponds to the second eigenvector, etc. Characteristics used are: market capitalization, volume traded, dividend yield, earnings per share. For each fundamental characteristic, values were mapped to $[0,1]$; negative values for components (on the left) indicate short positions.

\subsection{Interpretation of leading eigenvectors}

Several studies have investigated market segmentation or clustering via metrics obtained from correlation matrices ([21], [15], [16] and [3]). In [28], the authors are able to interpret eigenvalues deviating from the RMT noiseband in a similar way. In that investigation, because it was an order of magnitude greater than the rest, the effect of the leading eigenvalue was removed from the data by regressing each stock return times series against the leading eigenmode to obtain a new correlation matrix from stock specific return components (as in the Capital Asset Pricing Model). The new leading eigenvector exhibited significant contributions from about $1 / 3$ of the 999 stocks, all with large values for market capitalization. The next 9 eigenvectors all contained stocks belonging to distinct economic sectors. 
In this investigation, the leading eigenvalue for Case 3 was found to contribute much less significantly to the overall trace compared to the rest of the large eigenvalues. Moreover, its eigenvector components were not temporally stable, but displayed anti-correlations over time as the behaviour of market participants changed through the 1997 Russian GKO default, the 1998 emerging market contagion and the 2001 crash of the SA currency.

Since the leading eigenvectors could not be identified with distinct economic sectors, fundamental characteristics of each eigenvector were considered ${ }^{4}$. Eigenvector components were weighted according fundamental properties and particular attention was paid to eigenvectors corresponding to the largest eigenvalues. The fundamental properties considered were: market capitalization, volume traded, dividend yield and earnings per share. These variables were normalized and mapped to numbers between zero and unity. Economic sectors were interpreted in terms of spectra, ranging from resources, through industrial, non-cyclical and then cyclical shares into financial and then technology shares, as classified by the JSE, from smaller values to larger values and rescaled for the fundamental characteristic graphs.

This representation offers a method to inspect the differences between the compositions of the eigenmodes corresponding to different eigenvectors.

Figure 7 gives representations of the fundamental characteristics for eigenvectors corresponding to eigenvalues ranging from largest (bottom) to smallest (top of range). We include results for Cases 1 and 3 .

This analysis, together with the tests of temporal stability, suggests that the eigenmodes ${ }^{5}$ are not easily interpreted in terms of isolated characteristics. Instead eigenmodes may be viewed as being representative of distinct trading strategies prevalent in the market itself. This conclusion is motivated by the observation that negative component values of the eigenvectors imply shorting and the positive values imply long positions.

It can be deduced from Figure 7 that those eigenvectors which are associated with eigenvalues lying in the noise band seem to correspond to trading strategies which hold roughly equal long and short positions in mid-capitalization stocks. Case 1 and Case 3 are qualitatively the same in this sense for eigenvalues in the noise band.

For Case 1, the first eigenvector is long in large capitalization, large volume resource and industrial shares. The next leading eigenvector carries short positions in large capitalization, large volume shares. For all the eigenvectors in this case there is indistinct economic sector characterisation. This gives further evidence that there is information loss with zero padding and zero-order hold.

For Case 3 of Epoch 6, the leading eigenvector has similar long positions as in Case 1. The second eigenvector is quite different from the first for Case 3, as well as from its

4 Fundamental is used in the 'bottom up' sense of financial analysis, i.e. in terms of unique characteristics of stocks such as earnings, dividends, market capitalization, book value, etc...

5 The eigenmode for each eigenvector is the timeseries derived from the timeseries of the eigenvector components. Leading eigenmodes are also sometimes referred to as principal components. 


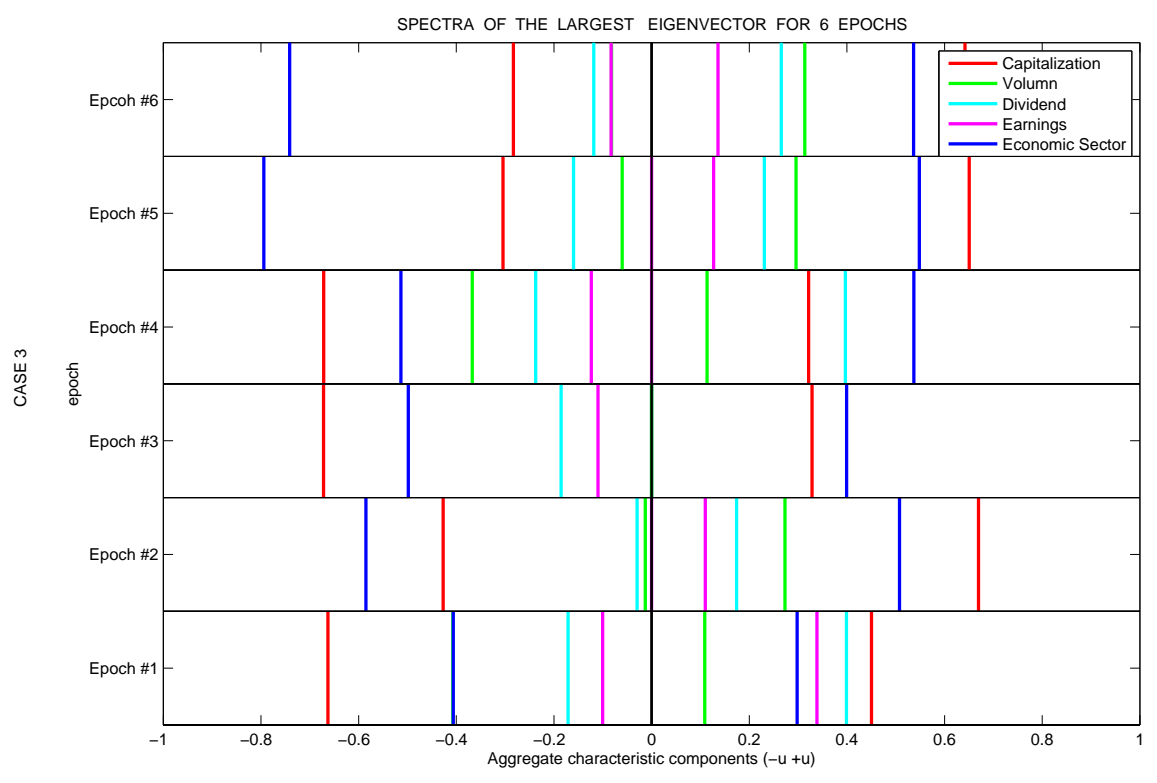

Fig. 8. Fundamental characteristics of components of the $1^{\text {st }}$ eigenvectors for the 6 epochs for Case 3, where Epochs 1 to 6 demarcate time windows 1993-1997 to 1998-2002 (cf. Figure 1).

counterpart in Case 1: t exhibits long positions in stocks with smaller capitalization and short positions in stocks with relatively larger capitalization; it is long in stocks with low earnings, low volume and low dividend yield. In general the leading eigenvectors in Case 3 have more varied compositions.

Small eigenvalues for Case 3 correspond to trading strategies which replicate long and short positions in small capitalization, low earnings and lower volume and dividend yield relative to the noise band. Cases 1 and 3 deviate markedly in this regard.

Similarly, inspection of the graph shows that characteristics for the rest of the eigenvectors are different in Cases 1 and 3. In the former, the characteristics seems to settle to a noisy composition sooner. This is consistent with findings for the inverse participation ratios.

In Figure 8 we compare the fundamental characteristics for the leading eigenmode for the different epochs. In Epoch 1, the $1^{\text {st }}$ eigenmode is long in comparatively higher earnings, smaller capitalization and low volume stocks, while it is short lower earnings and large capitalization stocks. In contrast, in Epoch 5 (1997-2001) and Epoch 6 (1998-2002), the $1^{\text {st }}$ eigenmodes are dominated by long positions in large capitalization stocks and short positions in small capitalization financial stocks, where the financial sector corresponds to a economic sector value of 0.8 . This depiction of a shift in the market is consistent with the historical context observed in Figure 1.

In Figure 8 the short positions in Epoch 5 and 6 offer the only occurrences where aggregate quantification of sector participation correlates uniquely with one sector. In general, there is always significant share concentration in the resources sector (sector value 0.0) for the period of investigation as well as varying activity in industrial (sector value 0.6) and financial stocks. As a result, eigenmodes are not differentiated by sector participation and 
in particular, the single aggregate quantifier for sector participation of eigenmodes considered in this investigation is generally ineffective. This is not surprising in an emerging market, where stock concentration, currency volatility and generally high co-movement with commodity prices blur economic sector independence. Inspection of these quantifiers for the second eigenmodes corroborates these findings.

\section{Conclusions}

Our investigation exposes some notable differences in the spectral properties of the correlation matrices computed by the three different methods outlined. As in preceding analyses of financial market data, in all cases we have found that the distribution of eigenvalues exhibits: (1) a significant part of the spectrum falls within the range of random matrix predictions, and (2) there exists a small no. of large leading eigenvalues. However, we found that by computing measured-data correlation matrices, Case 3, a far less substantial part of the spectrum falls within the Wishart range (Eqn. 1) than when computing correlations with zero padding, Case 1. Similar results were found when comparing the inverse participation ratios of Cases 1 and 3 with their RMT counterparts. Results for Case 2, which incorporated zero-order hold but not zero padding, varied with the RMT tests; here the eigenvector components and inverse participation ratios were closest to RMT predictions.

Our investigation suggests that zero padding and zero-order hold increases the level of noise in the estimation of correlation matrices. The correlations between leading eigenvectors from successive epochs also showed evidence of greater temporal stability when measured-data correlations were used.

Our fundamental characteristic investigation suggests that the leading eigenmodes may be interpreted in terms of independent trading strategies with long range correlations. These are more distinct for the measured-data correlation case than when there was zero padding and zero-order hold.

\section{Acknowledgements}

We thank the referees of Physica A for their pertinent comments and questions. DW thanks National Research Foundation Thuthuka Grant TTK2005081000005 and University of Cape Town Research Council for financial support.

\section{References}

[1] Z.D. Bai, Methodologies in spectral analysis of large dimensional random matrices, a review, Statistica Sinica, 9 (1999), 611-677 
[2] G. Bonanno, F. Lillo and R.N. Mantegna, High-frequency Cross-correlation in a Set of Stocks, Quantitative Finance, 1 (2001), 96-104, e-print cond-mat/0009350

[3] G. Bonanno, G. Caldarelli, F. Lillo, S. Miccichè, N. Vandewalle and R. N. Mantegna, Networks of equities in financial markets, Eur. Phys. J. B, 38 (2004), 363-371 e-print cond-mat/0401300

[4] J.-P. Bouchaud and M. Potters, Theory of Financial Risks - from statistical physics to risk management, Cambridge University Press, Cambridge, 2000

[5] T.A. Brody, J.Flores, J.B.French, P.A.Mello, A.Pandey and S.S.M.Wong, Random- matrix physics: spectrum and strength fluctuations, Rev.Mod. Phys., 53 (1981), No. 3, 385-480

[6] Z. Burda, A. Goerlich, A. Jarosz and J. Jurkiewicz, Signal and Noise in Correlation Matrix, e-print cond-mat/0305627

[7] Y. Chen and J.E. McInroy, Estimation of Symmetric Positive Definite Matrices from Imperfect Measurements IEEE Transactions on Automatic Control, Vol. 47, No. 10 (2002), $1721-1725$

[8] S. Drożdż, F. Grümmer, F.Ruf and J.Speth, Dynamics of competition between collectivity and noise in the stock market, Physica A, 287 (2000), 440- e-print cond-mat/9911168

[9] S. Drożdż, J. Kwapien, F. Grümmer, F. Ruf and J. Speth Quantifying dynamics of the financial correlations, Physica A, 299 (2001), 144- , e-print cond-mat/0102402

[10] S. Drożdż, J. Kwapien, J. Speth and M. Wojcik, Identifying Complexity by Means of Matrices, Physica A, 314 (2002), 355-361, e-print cond-mat/0112271

[11] A. Edelman, Eigenvalues and condition numbers of random matrices, SIAM J. Matrix Anal. Appl., 9 (1988), No. 4, 543-560

[12] E. Fama and K. French, The cross section of expected share returns, J. Finance, 47 (1992), 427-465

[13] S. Gallucio, J-P. Bouchaud and M. Potters, Rational decisions, random matrices and spin glasses, Physica A, 259 (1998), 449-456

[14] T. Guhr, A. Müller-Groeling and H.A. Weidenmüller, Random matrix theories in quantum physics: common concepts, Phys. Rep., 299 (1998), 190-

[15] L. Giada and M. Marsili, Data clustering and noise undressing of correlation matrices, Phys. Rev. E, 63 (2001) 061101-

[16] P. Gopikrishnan, B. Rosenow, V. Plerou and H. E. Stanley, Identifying Business Sectors from Stock Price Fluctuations, Phys. Rev. E, 64 (2001), 035106-

[17] L. Laloux, P. Cizeau, J.P. Bouchaud and M. Potters, Noise Dressing of Financial Correlation Matrices, Phys. Rev. Lett., 83 (1999), 1467-1470

[18] O. Ledoit and M. Wolf A Well-Conditioned Estimator for Large-Dimensional Covariance Matrices J. Multivariate Analysis, Vol. 88, Issue 2 (2004), 365-411

[19] F. Lindskog, Linear Correlation Estimation., e-print http://www.risklab.ch/Papers.html\#LCELindskog (2000) 
[20] R.N. Mantegna and H.E. Stanley, Introduction to Econophysics, - correlations and complexity in finance, Cambridge University Press, Cambridge, 2000

[21] R.N. Mantegna, Hierarchical Structure in Financial Markets, Eur. Phys. J. B, 11 (1998), 193-197

[22] H. Markowitz, Portfolio Selection: Efficient Diversification of Investments, Wiley, New York, 1959

[23] M.L. Mehta, Random Matrices, 2nd edition Academic Press, New York, 1991

[24] J.D. Noh, Model for correlations in stock markets, Phys. Rev. E, 61 (2000), 5981-

[25] S. Pafka and I. Kondor, Noisy covariance matrices and portfolio optimisation II, Physica A, 319 (2003), 487-494, e-print cond-mat/0205119

[26] S. Pafka and I. Kondor, Estimated correlation matrices and portfolio optimization, Physica A, 343 (2004), 623-634

[27] V. Plerou, P. Gopikrishnan, B. Rosenow, L.A.N. Amaral and H.E. Stanley, Universal and non-universal properties and cross correlations in financial time series, Phys. Rev. Lett., $\mathbf{8 3}$ (1999), 1471-1474

[28] V. Plerou, P. Gopikrishnan, B. Rosenow, L.A.N. Amaral, T. Guhr and H. E. Stanley, Random matrix approach to cross correlations in financial data, Phys. Rev. E, 65 (2001), 066126-

[29] D. Polakow and T. Gebbie, How many independent bets are there?, e-print cond-mat/0601166

[30] B. Rosenow, P. Gopikrishnan, V. Plerou and H. E. Stanley, Dynamics of cross-correlations in the stock market, Physica A, 324 (2003), 241-246

[31] A.M. Sengupta and P.P. Mitra, Distribution of Singular Values for Some Random Matrices, Phys. Rev. E, 60 (1999), 3389-

[32] D.Wilcox and T. Gebbie, Periodicity and scaling of eigenmodes in an emerging market, e-print cond-mat/0404416

[33] W. Wothke, Longitudinal and multi-group modeling with missing data, in Modeling longitudinal and multilevel data: Practical issues, applied approaches and specific examples, T.D. Little, K.U. Schnabel and J. Baumert [Eds.], Lawrence Erlbaum Associates, Mahwah, NJ, 2000

[34] Missing Data Methods, http://www.secondmoment.org/etal-column/ea-missingdata.php 